\title{
Sleep-Related Cognitive/Behavioral Predictors of Sleep Quality and Relapse in Individuals with Alcohol Use Disorder
}

\author{
Alyssa Todaro Brooks ${ }^{1} \cdot$ Narjis Kazmi $^{1} \cdot$ Li Yang $^{1} \cdot$ Ralph Thadeus Tuason ${ }^{1} \cdot$ Michael Charles Krumlauf ${ }^{1}$. \\ Gwenyth Reid Wallen ${ }^{1}$
}

Published online: 27 May 2020

(C) The Author(s) 2020

\begin{abstract}
Background Little is known about cognitive and behavioral predictors of sleep quality and relapse among individuals with alcohol use disorder (AUD). Using the social cognitive theory (SCT), we assessed sleep-related behaviors and cognitions, sleep quality, and relapse to drinking among individuals with AUD transitioning from inpatient to outpatient settings.

Method Individuals $(n=149)$ seeking treatment for AUD were recruited during their inpatient stay. Self-efficacy for sleep, dysfunctional beliefs about sleep, sleep-related behaviors, sleep quality, and relapse were assessed. Objective (actigraphy) assessment of sleep efficiency and duration was measured using actigraphy. Multiple logistic regression models tested whether self-reported sleep quality or sleep-related beliefs/behavior predicted relapse. Repeated measures linear mixed modeling tested whether there was a change over time in sleep quality as well as the relationships between self-efficacy, sleep-related beliefs, sleep behaviors, sleep quality, and relapse.

Results In our sample, self-efficacy for sleep, dysfunctional beliefs about sleep, and sleep-related behavior were all significantly associated with both sleep quality and relapse. Controlling for pre-discharge sleep-related behaviors (SRBQ) and actigraphyrecorded average sleep time during the first week post-discharge, married participants had lower odds of relapse compared with non-married patients $(p=0.048, \mathrm{OR}=0.119,95 \%$ CI $0.015-0.983)$. Patients with lower self-efficacy for sleep (SES) scores $(p<0.001)$ and higher CPRS anxiety scores $(p<0.001)$ had higher PSQI scores.

Conclusion Our results highlight the importance of self-efficacy and dysfunctional beliefs about sleep as predictors of sleep quality and relapse among individuals with AUD and the utility of the SCT as a sleep research framework.
\end{abstract}

Keywords Alcohol use disorder $\cdot$ Insomnia $\cdot$ Sleep disturbance $\cdot$ Cognitive behavioral therapy for insomnia $\cdot$ alcohol $\cdot$ sleep

\section{Background}

Alcohol use disorder (AUD; formerly alcohol "abuse" or "dependence") is often accompanied by various psychiatric and socio-behavioral comorbidities, including significant sleep disturbances [1-3]. Alcohol can negatively affect many aspects of sleep including the proportion of rapid eye movement

Electronic supplementary material The online version of this article (https://doi.org/10.1007/s12529-020-09901-9) contains supplementary material, which is available to authorized users.

Alyssa Todaro Brooks todaroad@mail.nih.gov

1 Nursing Research and Translational Science, National Institutes of Health Clinical Center, 10 Center Drive Room 2B13, Bethesda, MD 20892, USA
(REM) sleep, nightmare frequency, sleep fragmentation, and snoring. These effects manifest in varying intensity through stages of drinking, withdrawal, and abstinence [4, 5]. Multiple studies have reported a prevalence of insomnia symptoms ranging from 36 to $91 \%$ among patients with alcohol dependence [6]. Ford and Kamerow [7] demonstrated that individuals who met criteria for alcohol abuse and dependence were more likely to report ever experiencing a period of two or more weeks of insomnia when compared with non-alcoholdependent individuals.

Individuals may use alcohol to self-medicate sleep disturbance. Those with sleep disturbances may choose to drink alcohol specifically because of its depressive effects [8], and individuals with insomnia may be more likely to self-medicate with alcohol [9]. Heavy alcohol consumption can induce fatigue and reduce sleep onset latency thereby speeding up the process of falling asleep [10-12], which may be particularly tempting for those who are struggling with difficulty sleeping 
and already accustomed to drinking heavily. Recently, Roehrs and Thomas [13] uncovered the risk associated with using alcohol as a sleep aid; as tolerance develops, this results in self-administration of significantly higher amounts of alcohol for sleep. Specifically among alcohol-dependent individuals, re-initiation of drinking following abstinence may be an attempt to self-medicate sleep problems $[12,14,15]$. Kolla and colleagues [14] reported that in a sample of individuals who were alcohol dependent admitted for a 30-day addiction treatment program, more than half $(51 \%)$ self-reported the use of alcohol to help them fall asleep and this use of alcohol significantly predicted relapse over a period of 12 months. Similarly, in another mixed methods study assessing sleeprelated beliefs and behaviors of treatment-seeking AUD patients, participants reported the use of alcohol to self-medicate for sleep and anxiety [16].

Sleep disturbance may be associated with relapse to drinking. Among individuals who seek treatment, baseline sleep problems upon entering treatment may predict subsequent relapse to drinking [17-21]. Smith and colleagues [20] demonstrated that longer sleep onset latency during inpatient rehabilitation predicted relapse within one month of discharge from the treatment facility. Self-reported use of alcohol to fall asleep is associated with relapse over 12 months following a one-month residential treatment [14]. Individuals who report insomnia within six months prior to achieving abstinence are more likely to relapse after five months of abstinence [22].

Theoretical framework Both AUD and sleep disturbances are complex, multi-factorial, and impact multiple facets of individuals' lives. We chose to use the social cognitive theory (SCT) to examine the relationship between sleep and relapse. The SCT has been widely used to predict and modify behavior, including abstinence [23]. The SCT posits that personal factors, the environment, and human behavior exert influence upon each other through reciprocal determinism [24]. A recent review called for theoretical frameworks to be used when assessing sleep, in order to better understand behavior [25]. Sleep-related cognitions, thoughts, and perceptions and sleeprelated behaviors should be carefully considered in maximizing the success of recovery efforts. Unhealthy sleep-related cognitions are established contributors to poor sleep [26, 27]. Cognitive arousal and inaccurate beliefs about sleep can lead to maladaptive sleep behaviors [27]. Behaviors such as late-night physical activity, daytime napping, and sleeping in on weekends can be harmful to overall sleep quality [27]. Sleep-related expectations and behavior are both influenced by an individual's sleeping environment: bedtime, lighting, temperature, pressure to attend to other obligations, bedpartner snoring, pets in the bedroom, and other factors. Lastly, a stronger belief in one's ability to achieve better sleep ("self-efficacy for sleep") may determine whether the response is a harmful sleep behavior or a healthier adaptation
[24]. Preliminary findings in a small cohort $(n=95)$ of the current study by Brooks and colleagues [16] showed significant improvements in self-efficacy for sleep over the course of inpatient treatment through discharge. Despite potential relevance for assessing/improving sleep health, the SCT (in its entirety) has "been applied to sleep research only in the context of adherence to medical sleep disorder treatments" [25](p. 7) and thus represents a novel approach to examining sleeprelated outcomes. Considering the importance of self-efficacy, outcome expectancies, and environment during transition from treatment in AUD as it relates to sleep, the SCT provided our theoretical framework to evaluate the potential relationships between sleep and relapse proposed in this study.

Purpose of study Little is known about cognitive and behavioral predictors of sleep quality and relapse among individuals with AUD. In the present study, we assessed sleep-related behaviors and cognitions, sleep quality (subjective and objective [actigraphy]), and relapse to drinking among individuals with AUD across the transition from an inpatient to outpatient setting. We also assessed basic demographic factors, including marital status, which could have implications for postdischarge environment/social support [28]. Our primary outcomes included sleep quality and relapse. We hypothesized the following: (1) higher self-efficacy for sleep would be associated with better sleep quality and lower relapse rates; (2) fewer dysfunctional beliefs about sleep would be associated with better sleep quality and lower relapse rates; (3) higher endorsement of sleep-related safety behaviors would be associated with poorer sleep quality and higher relapse rates; and (4) better sleep quality would be associated with lower relapse rates.

\section{Methods}

We recruited individuals $(n=149)$ seeking inpatient treatment for AUD initially enrolled on a screening and assessment clinical trial (NCT\#02231840). All participants received continued physical evaluations, inpatient treatment of alcohol withdrawal, psychosocial management, and an educational treatment program during their participation in this study. Participants were eligible for our study examining sleep disturbance throughout rehabilitation (NCT\#02181569) if they were 18 years of age or older, an inpatient for 21 days or more preceding discharge, not enrolled onto a pharmacologic intervention study, able to understand the study, and willing to complete a follow-up visit in-person or by phone four to six weeks after being discharged from inpatient treatment. The analysis described herein represents a sub-analysis of the total sample of 198 participants and includes only individuals who provided data on sleep quality/relapse post-discharge. All participants provided written informed consent. 
We used basic demographic, clinical, and alcohol-related variables to characterize the sample upon admission, including age, gender, marital status, race, ethnicity, the Structured Clinical Interview for DSM IV or DSM 5 diagnosis (SCID; [29]), alcohol drinking history before admission using Timeline Follow-back (TLFB; [30, 31]), alcohol craving using the Penn Alcohol Craving Scale (PACS; [32]), severity of alcohol withdrawal using the Clinical Institute Withdrawal Assessment (CIWA; [33]), and depression and anxiety using two subscales of the Comprehensive Psychopathological Rating Scale (CPRS; [34-36]); brief scale for anxiety (BSA) and Montgomery-Asberg depression rating scale (MADRS).

Our primary outcome of sleep quality was self-reported (Pittsburgh Sleep Quality Index-PSQI; [37-39]). We also assessed excessive daytime sleepiness (Epworth Sleepiness Scale-ESS; [40-43]), dysfunctional beliefs/attitudes about sleep (Dysfunctional Beliefs and Attitudes about Sleep Scale, DBAS-16; [44]), self-efficacy for sleep (Self-Efficacy for Sleep Scale, SE-S; [45-47]), and sleep-related behaviors (Sleep-Related Behaviors Questionnaire, SRBQ; [48]). Refer to Supplemental Table 1 for a description of all measures and the timing of their administration.

\section{Objective measure of sleep: Respironics Actiwatch Spectrum}

Plus "Actiwatches" are small actigraphy-based wristband data loggers that record digitally integrated measures of gross motor activity. These devices contain accelerometers and light sensors in order to objectively assess sleep and activity. Prior studies have demonstrated actigraphy's high sensitivity with moderate accuracy for assessing sleep parameters in populations with normal and disturbed sleep when compared with polysomnography [49-51]. The Actiwatch Spectrum Plus provided a battery life adequate for the outpatient research phase of this protocol. The watch is worn on the nondominant wrist. For approximately one week before their scheduled discharge date, patients wore an Actiwatch Spectrum Plus (Philips Respironics) up until four weeks from their date of discharge, which coincided with the final visit for follow-up surveys. Main outcomes from actigraphy included weekly averages of sleep efficiency, wake after sleep onset, and time in bed.

Sleep/relapse diary We used daily sleep and symptom diaries to cross-validate objective sleep data collected via actigraphy and assess additional symptoms not captured by other assessments. We assessed relapse from patient report of alcohol consumption in the diary (primary outcome) and asked patients to add up the number of alcoholic drinks consumed per day. Patients were instructed to complete diaries once daily (in the morning upon waking).

Study timeline overview Approximately one week prior to patients' scheduled discharge, a study team member approached patients to begin the first segment of data collection for the study. Participants completed assessments of daytime sleepiness, self-efficacy for sleep, and sleep-related beliefs and behaviors at this time. Four to six weeks following discharge, participants completed a follow-up visit in-person or by phone.

Actigraphy analyses After device removal and data download, raw data from the Actiwatch Spectrum Plus were analyzed using the Respironics computerized sleep scoring software, which scores each epoch based on a threshold method algorithm. Investigators reviewed each sleep period prior to analysis to screen for malfunctioning watches, corrupt data, and required adjustments using bedtimes and wake times from the diary self-reports when necessary.

Statistical analysis All data were double-data entered, crosschecked, and reconciled as necessary. We dichotomized marital status (married vs. non-married) and used the last possible CPRS scores prior to discharge (day 23 or 30 depending on patients' length of stay). For withdrawal scores, we used the overall maximum score of any day of treatment. We treated relapse as a dichotomous variable; any drinking within four weeks of discharge from inpatient treatment was considered relapse based on diary self-report. Relationships between variables were examined with bivariate correlation coefficients (for two continuous variables), chi-squares (for two categorical variables), and basic $t$ tests (for one categorical and one continuous variable). All variables associated with relapse with $p$ values less than 0.20 were included in the multiple logistic regression model to test whether sleep quality or sleep-related beliefs/behavior predict relapse. Backward stepwise with an entering criterion of 0.05 and a removal criterion of 0.10 to select the variables in the final model was used. Repeated measures linear mixed modeling was performed to test whether there was a change over time in sleep quality as well as the relationships between sleep quality and relapse, self-efficacy, sleep-related beliefs, and sleep behaviors. A restricted maximum likelihood (REML) procedure for model parameter estimation was utilized. We used Aikake information criterion (AIC) and Bayesian information criterion (BIC) to compare and select models. All data analyses were conducted using IBM SPSS statistics and SAS 9.4. A $p$ value less than 0.05 was considered statistically significant.

\section{Results}

Study participants $(n=149)$ were mostly male $(n=99,66.4 \%)$ and not married $(n=119,79.9 \%)$. Of the 119 unmarried individuals, $21(14.1 \%)$ were divorced, seven $(4.7 \%)$ were separated, $88(59.1 \%)$ were single, three $(2.0 \%)$ were widowed, and three $(2.0 \%)$ did not report a marital status. We compared 
demographic and clinical variables between those who relapsed and those who did not relapse (Table 1 and Table 2). Those who relapsed were younger $(44.97 \pm 12.75$ vs. $49.59 \pm$ $10.18, p=0.045)$, less likely to be married (3.3\% vs. $26.4 \%$, $p=0.004)$, more likely to report higher levels of postdischarge craving (PACS score of $13.04 \pm 7.62$ vs. $7.11 \pm$ $6.08, p<0.01)$, more likely to self-report sleep disturbance pre-discharge (mean PSQI score of $9.0 \pm 3.10$ vs. $7.27 \pm$ $4.11, p=0.017)$, had lower self-efficacy for sleep postdischarge ( $26.32 \pm 7.05$ vs. $29.89 \pm 7.59, p=0.035)$, selfreported more dysfunctional beliefs about sleep before discharge $(4.70 \pm 1.68$ vs. $3.81 \pm 1.96, p=0.027)$, and engaged in more sleep-related behaviors (SRBQ score $52.73 \pm 14.22$ vs. $43.66 \pm 15.73 p<0.01$ and $51.36 \pm 20.21$ vs. $42.14 \pm$
Table 1 Demographic and clinical variables by relapse groups $(N=149)$

\begin{tabular}{|c|c|c|c|c|}
\hline Demographics $n=149$ & $\begin{array}{l}\text { Total sample } \\
n=149\end{array}$ & Relapse $n=30$ & $\begin{array}{l}\text { No relapse } \\
n=93\end{array}$ & $\begin{array}{l}p \\
\text { value }\end{array}$ \\
\hline Age, mean (SD) & $47.30(11.64)$ & $44.97(12.75)$ & $49.59(10.18)$ & 0.045 \\
\hline \multicolumn{4}{|l|}{ Gender, $n(\%)$} & \multirow[t]{3}{*}{0.27} \\
\hline Male & $99(66.4)$ & $17(56.67)$ & $64(68.82)$ & \\
\hline Female & $50(33.6)$ & $13(43.33)$ & $29(31.18)$ & \\
\hline \multicolumn{4}{|l|}{ Marital status } & \multirow[t]{3}{*}{0.004} \\
\hline Married & $27(18.5)$ & $1(3.3)$ & $24(26.4)$ & \\
\hline Single/widowed/divorced/separated & $119(79.9)$ & $29(96.7)$ & $67(73.6)$ & \\
\hline \multicolumn{4}{|l|}{ Race $(\%)$} & \multirow[t]{4}{*}{0.239} \\
\hline White & $81(54.4)$ & $17(56.67)$ & $52(55.91)$ & \\
\hline Black & $51(34.2)$ & $7(23.33)$ & $32(34.41)$ & \\
\hline Other & $17(11.4)$ & $6(20.0)$ & $9(9.68)$ & \\
\hline \multicolumn{4}{|l|}{ Ethnicity $(\%)$} & \multirow[t]{4}{*}{0.308} \\
\hline Not Hispanic or Latino & $137(91.9)$ & $26(86.7)$ & $86(93.5)$ & \\
\hline Hispanic or Latino & $10(6.7)$ & $4(13.3)$ & $5(5.4)$ & \\
\hline Unknown & $1(0.7)$ & 0 & $1(1.1)$ & \\
\hline \multicolumn{5}{|l|}{$\mathrm{SCID}-\mathrm{IV} / 5^{\mathrm{a}}, n=148$} \\
\hline One or more anxiety disorders $(\%)$ & $44(29.53)$ & $11(36.7)$ & $26(28.3)$ & 0.493 \\
\hline One or more mood disorders $(\%)$ & $45(29.53)$ & $11(36.7)$ & $27(29.3)$ & 0.499 \\
\hline \multicolumn{5}{|c|}{ Timeline Follow-back (90 days preceding admission) $N=145$} \\
\hline \multicolumn{5}{|l|}{ Mean (SD) } \\
\hline Average drinks/day, $n=145$ & $15.31(8.48)$ & $15.24(8.17)$ & $15.56(8.52)$ & 0.857 \\
\hline Number of drinking days, $n=145$ & $73.74(23.26)$ & $78.27(16.85)$ & $73.04(25.67)$ & 0.206 \\
\hline Number of heavy drinking days, $n=145$ & $70.32(26.05)$ & $75.70(21.77)$ & $68.99(28.59)$ & 0.183 \\
\hline \multicolumn{5}{|l|}{ Penn Alcohol Craving Scale } \\
\hline \multicolumn{5}{|l|}{ Mean (SD) } \\
\hline Day 5 of inpatient admission, $n=147$ & $10.74(7.37)$ & $11.40(7.59)$ & $10.82(7.49)$ & 0.712 \\
\hline Post-discharge, $n=111$ & $8.35(6.91)$ & $13.04(7.62)$ & $7.11(6.08)$ & 0.000 \\
\hline $\begin{array}{l}\text { Clinical Institute Withdrawal Assessment } \\
\text { (CIWA) }\end{array}$ & $8.52(5.96)$ & $10.20(8.11)$ & $8.11(4.84)$ & 0.19 \\
\hline \multicolumn{5}{|c|}{$\begin{array}{l}\text { Max score mean (SD) } \\
\text { Comprehensive Psychopathology Rating Scale (CPRS) }\end{array}$} \\
\hline \multicolumn{5}{|l|}{ Brief Scale for Anxiety (BSA), mean (SD) } \\
\hline Day 2 of inpatient admission, $n=148$ & $12.72(6.69)$ & $14.28(5.98)$ & $12.0(6.77)$ & 0.107 \\
\hline Pre-discharge, $n=149$ & $5.22(4.70)$ & $5.90(4.75)$ & $5.12(4.63)$ & 0.426 \\
\hline \multicolumn{5}{|c|}{ Montgomery-Åsberg Depression Rating Scale (MADRS) mean (SD) } \\
\hline Day 2 of inpatient admission, $n=148$ & $16.72(8.61)$ & $18.83(8.90)$ & $16.01(8.60)$ & 0.129 \\
\hline Pre-discharge, $n=149$ & $5.21(5.52)$ & $6.37(6.12)$ & $5.22(5.53)$ & 0.336 \\
\hline
\end{tabular}

a 82 participants were assessed with the DSM SCID-IV and 66 were assessed with DSM SCID-5

${ }^{\mathrm{b}}$ Depending on participants' length of stay, last available scores before discharge were used for pre-discharge BSA and MADRS; $n=99$ from day 30 and $n=50$ from day 23 
Table 2 Sleep measures by relapse status $(N=149)$

\begin{tabular}{|c|c|c|c|c|}
\hline $\begin{array}{l}\text { Sleep measures } \\
\text { Mean (SD) }\end{array}$ & Total sample $n=149$ & Relapse $n=30$ & No relapse $n=93$ & $p$ value* \\
\hline \multicolumn{5}{|c|}{ Pittsburgh Sleep Quality Index (PSQI) } \\
\hline Pre-discharge, $n=148$ & $7.74(3.93)$ & $9.00(3.10)$ & $7.27(4.11)$ & 0.017 \\
\hline Post-discharge, $n=109$ & $7.28(3.85)$ & $8.64(3.19)$ & $7.03(3.98)$ & 0.067 \\
\hline \multicolumn{5}{|l|}{ Self-Efficacy for Sleep (SE-S) } \\
\hline Pre-discharge, $n=148$ & $28.55(6.66)$ & $27.23(5.22)$ & $28.83(7.09)$ & 0.19 \\
\hline Post-discharge, $n=111$ & $29.19(7.67)$ & $26.32(7.05)$ & $29.89(7.59)$ & 0.035 \\
\hline \multicolumn{5}{|c|}{ Dysfunctional Beliefs and attitudes about Sleep (DBAS) } \\
\hline Pre-discharge, $n=149$ & $3.96(1.98)$ & $4.70(1.68)$ & $3.81(1.96)$ & 0.027 \\
\hline Post-discharge, $n=111$ & $3.81(2.10)$ & $4.02(2.02)$ & $3.86(2.12)$ & 0.739 \\
\hline \multicolumn{5}{|l|}{ Epworth Sleepiness Scale (ESS) } \\
\hline Pre-discharge, $n=149$ & $6.33(4.05)$ & $6.53(4.02)$ & $5.89(4.16)$ & 0.455 \\
\hline Post-discharge, $n=111$ & $5.59(4.03)$ & $5.16(3.60)$ & $5.78(4.18)$ & 0.507 \\
\hline \multicolumn{5}{|c|}{ Sleep-Related Behaviors Questionnaire (SRBQ) } \\
\hline Baseline/pre-discharge $n=149$ & $45.01(16.56)$ & $52.73(14.22)$ & $43.66(15.73)$ & 0.006 \\
\hline 4 weeks post-discharge $n=111$ & $43.44(19.83)$ & $51.36(20.21)$ & $42.14(19.03)$ & 0.039 \\
\hline \multicolumn{5}{|l|}{ Actigraphy variables } \\
\hline \multicolumn{5}{|l|}{ Sleep duration } \\
\hline Baseline week $1 n=120$ & $433.59(89.87)$ & $411.51(107.50)$ & $438.40(84.60)$ & 0.173 \\
\hline Week 4 post-discharge $n=106$ & $442.19(91.73)$ & $425.64(99.27)$ & $447.29(89.22)$ & 0.229 \\
\hline \multicolumn{5}{|l|}{ Sleep efficiency } \\
\hline Baseline week $1 n=120$ & $80.22(8.18)$ & $78.54(9.68)$ & $81.13(7.45)$ & 0.141 \\
\hline $\begin{array}{l}\text { Week } 4 \text { Post-discharge } \\
n=106\end{array}$ & $80.22(9.23)$ & $80.99(11.86)$ & $80.08(8.41)$ & 0.669 \\
\hline \multicolumn{5}{|c|}{ Wake after sleep onset (WASO) minutes } \\
\hline Baseline week $1 n=120$ & $60.05(21.90)$ & $61.38(20.35)$ & $57.55(20.10)$ & 0.381 \\
\hline Week 4 post-discharge $n=106$ & $61.37(29.01)$ & $53.73(23.19)$ & $63.26(29.16)$ & 0.139 \\
\hline \multicolumn{5}{|l|}{ Sleep onset latency } \\
\hline Baseline week $1 n=120$ & $15.78(16.43)$ & $14.03(16.72)$ & $16.06(16.63)$ & 0.574 \\
\hline Week 4 post-discharge $n=106$ & $18.22(19.17)$ & $14.74(16.31)$ & $18.92(19.63)$ & 0.338 \\
\hline \multicolumn{5}{|l|}{ Total sleep time } \\
\hline Baseline week $1 n=120$ & $372.63(84.43)$ & $348.84(102.42)$ & $380.11(77.90)$ & 0.09 \\
\hline Week 4 post-discharge $n=106$ & $379.87(83.21)$ & $371.01(100.91)$ & $383.04(78.19)$ & 0.589 \\
\hline
\end{tabular}

* $p$ values reflect comparison between relapse groups
19.03, $p=0.04$ respectively) at both time points. For both individuals who relapsed and those who did not relapse, participants' weekly average sleep duration improved from week 1 to week 4 (Table 2), but there were no significant differences between relapse status groups on any actigraphy variables at either time point.

Refer to Fig. 1 for a flow diagram outlining participant recruitment and retention. Since the two primary outcomes included sleep quality (PSQI) and relapse, we assessed differences between those who completed each of the aforementioned assessments and those who did not. There were no significant gender, race, ethnicity, or marital status differences between those who provided $(n=123)$ and who did not provide $(n=26)$ relapse data. Those who did not provide relapse data for 28 days post-discharge were significantly younger (41.8 vs. $48.5, p=0.007)$. There were no significant differences between those who provided $(n=109)$ and who did not provide $(n=40)$ PSQI data. Those who did not provide PSQI data 4-6 weeks post-discharge had significantly higher actigraphyrecorded mean wake bouts during week 1 (28.9 vs. 24.3, $p=0.017)$.

Results of hypothesis testing. Hypothesis 1 Higher selfefficacy for sleep (SE-S) is associated with better sleep quality and lower relapse rates. For both time points, higher SE-S was 
Fig. 1 Flow diagram outlining participant recruitment and retention

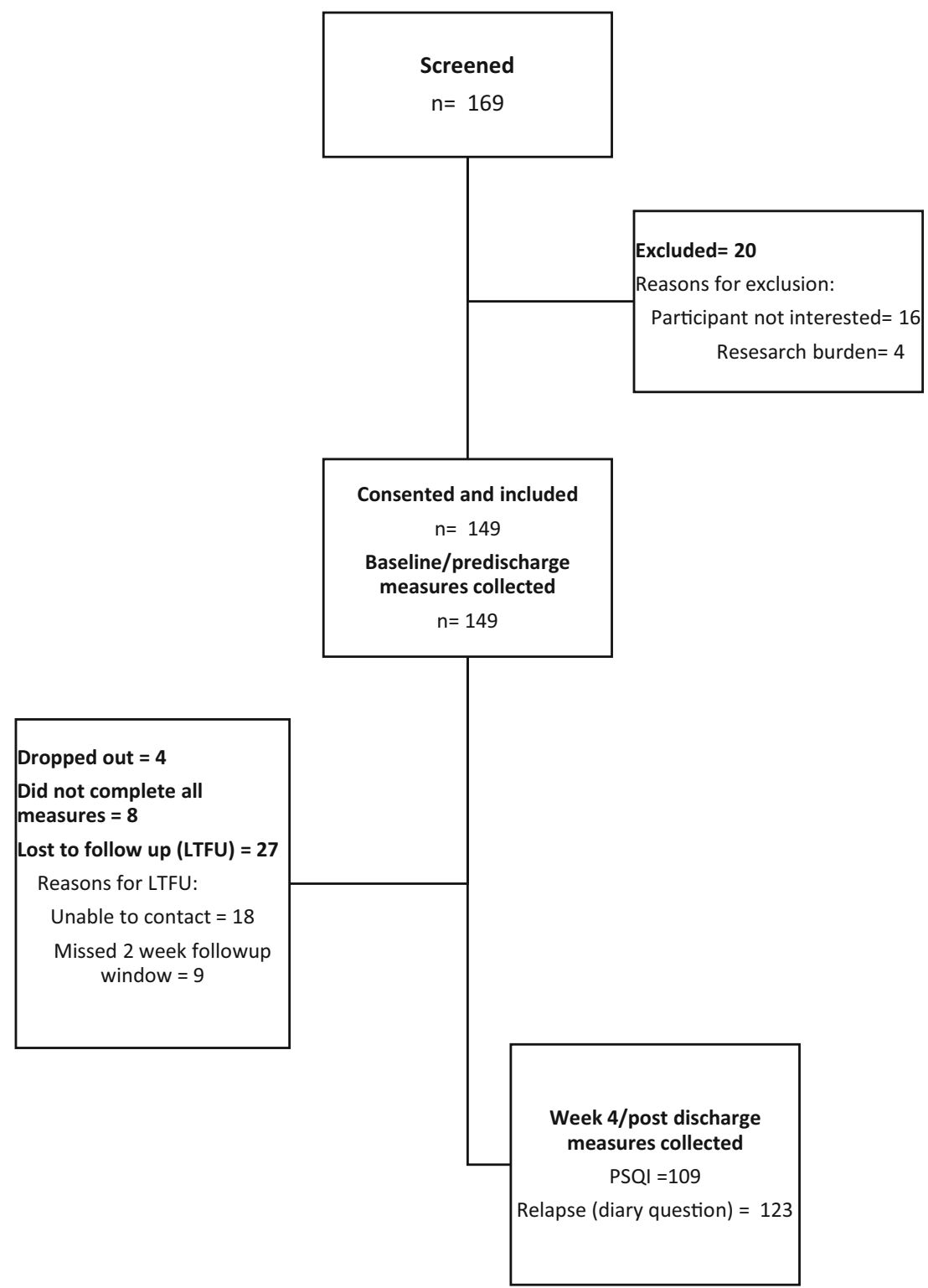

associated with a lower PSQI score $(p<0.001)$. No significant differences were found in pre-discharge self-efficacy scores between participants who relapsed $(27.23 \pm 5.22)$ and participants who did not relapse $(28.83 \pm 7.09, p=0.19)$.

Hypothesis 2 Fewer dysfunctional beliefs and attitudes about sleep (DBAS) was associated with better sleep quality and lower relapse rates. For both time points, a higher DBAS score was associated with higher PSQI scores $(p<0.001)$. Patients who relapsed had significantly higher week 1 DBAS scores $(4.70 \pm 1.68$ vs. $3.81 \pm 1.96, p=0.027)$.

Hypothesis 3 Higher endorsement of sleep-related safety behaviors (SRBQ) is associated with poorer sleep quality and higher relapse rates. For both time points, higher SRBQ scores were associated with higher PSQI scores $(p<0.001)$. Patients who relapsed had significantly higher week 1 SRBQ scores $(52.73 \pm 14.22$ vs. $43.66 \pm 15.73, p=0.006)$.

Hypothesis 4 Better sleep quality is associated with lower relapse rates. Individuals who relapsed had higher predischarge PSQI scores $(9.0 \pm 3.1$ vs. $7.27 \pm 4.11, p=$ 0.017).

Model 1: relapse In the final model of relapse (Table 3), controlling for pre-discharge sleep-related behaviors (SRBQ) and actigraphy-recorded average sleep time during the first week post-discharge, married patients had lower odds of relapse compared with non-married patients $(p=0.048, \mathrm{OR}=0.119$, 95\% CI 0.015-0.983). Controlling for marital status and actigraphy-recorded average sleep time during the first week post-discharge, patients with higher sleep-related behaviors 
Table 3 Predictors of relapse

\begin{tabular}{llllll}
\hline Predictors & \multicolumn{1}{c}{ Beta } & Std. Error & Wald-statistic & 95\% CI & $p$ value \\
\hline Married & -2.125 & 1.075 & 3.904 & $0.015-0.983$ & 0.048 \\
Sleep-related behaviors (SRBQ) $^{\mathrm{a}}$ & 0.045 & 0.020 & 4.987 & $1.006-1.088$ & 0.026 \\
Total sleep time $^{\mathrm{b}}$ & -0.006 & 0.003 & 3.744 & $0.987-1.000$ & 0.053 \\
\hline
\end{tabular}

Variable(s) entered in the logistic regression model were age, married, baseline/pre-discharge DBAS, PSQI, SRBQ, sleep efficiency, sleep time, SE-S, post-discharge PSQI, and MAX CIWA days 1-4

${ }^{a}$ Baseline/pre-discharge data

${ }^{\mathrm{b}}$ Average from week 1 (post-discharge) of actigraphy

scores had higher odds of relapse $(p=0.026, \mathrm{OR}=1.046$, 95\% CI 1.006-1.088).

Model 2: sleep quality (PSQI) There were no significant changes in PSQI scores from pre- to post-discharge. In the model predicting sleep quality (Table 4), controlling for all other variables in the model, patients with lower self-efficacy for sleep (SES) scores $(p<0.001)$ and higher pre-discharge CPRS anxiety scores $(p<0.001)$ had higher PSQI scores.

\section{Discussion}

Results of the current study highlight the importance of cognitive and behavioral predictors of sleep quality and relapse among individuals with AUD. In our sample, self-efficacy for sleep, dysfunctional beliefs about sleep, and sleep-related behavior were all significantly associated with both sleep quality and relapse. Additionally, sleep quality was associated with relapse. Similar to previous findings [17-21], our results further describe that among treatment-seeking individuals, baseline sleep disturbances at the start of transition from inpatient to outpatient environment may predict relapse to drinking. This evidence makes the case for improving sleep quality as an important target for comprehensive and targeted treatment among individuals with AUD.

The role of marital status in predicting relapse was an interesting finding and further supports our previous qualitative work [16] in the same population, which pointed to social support as a potentially important variable in sustained sobriety. These results support the need for future research that considers post-discharge social support more broadly. Understanding the immediate support network following inpatient treatment, including spouses and other family members as well as their drinking patterns and the type of support they provide, could help researchers and clinicians customize treatment plans.

In our sample, there were no statistically significant differences in actigraphy-recorded variables between those who relapsed and those who did not relapse. Instead, differences between the cognitive and behavioral variables (specifically self-efficacy for sleep, dysfunctional beliefs and attitudes about sleep, and sleep-related behaviors) were more pronounced between the two groups.

As in other studies $[15,52]$, we found that even in those who did not relapse, and whose sleep quality improved, sleep disturbance persisted post-abstinence. This persistence of sleep disturbances has important implications for how interventions such as cognitive behavioral therapy for insomnia
Table 4 Predictors of sleep quality

\begin{tabular}{|c|c|c|c|c|c|}
\hline Predictors & Estimate & $\begin{array}{l}\text { Std. } \\
\text { Error }\end{array}$ & $\begin{array}{l}t- \\
\text { statistic }\end{array}$ & $95 \% \mathrm{CI}$ & $\begin{array}{l}p \\
\text { value }\end{array}$ \\
\hline No relapse & -1.05 & 0.54 & -1.948 & $\begin{array}{c}-2.122 \text { to } \\
0.019\end{array}$ & 0.054 \\
\hline $\begin{array}{l}\text { Dysfunctional Beliefs and Attitudes about Sleep } \\
\text { (DBAS) }^{\mathrm{a}}\end{array}$ & 0.23 & 0.12 & 1.857 & $\begin{array}{c}-0.014 \text { to } \\
0.470\end{array}$ & 0.065 \\
\hline Self-Efficacy for Sleep (SE-S) ${ }^{a}$ & -0.18 & 0.04 & -4.741 & $\begin{array}{c}-0.248 \text { to } \\
0.102\end{array}$ & 0.000 \\
\hline Sleep onset latency ${ }^{\mathrm{a}}$ & 0.01 & 0.01 & 0.874 & $\begin{array}{c}-0.013 \text { to } \\
0.034\end{array}$ & 0.383 \\
\hline Max CIWA days $1-4$ & 0.06 & 0.04 & 1.595 & $\begin{array}{c}-0.015 \text { to } \\
0.146\end{array}$ & 0.114 \\
\hline CPRS anxiety pre-discharge & 0.23 & 0.05 & 4.219 & $\begin{array}{r}0.121 \text { to } \\
0.335\end{array}$ & 0.000 \\
\hline
\end{tabular}

${ }^{\text {a }}$ Longitudinal data from both timepoints was entered in the model 
(CBT-I) might be an important complement for therapy focused on sobriety [53]. Furthermore, our results emphasize the importance of improving self-efficacy related to sleep, addressing anxiety, and establishing healthy beliefs and behaviors related to sleep to improve sleep quality and increase the likelihood of sobriety.

Strengths and limitations The use of the SCT to ground our measures and analyses and identify cognitions/behaviors associated with sleep quality and relapse is a strength of this analysis; to our knowledge, it has not previously been used to explore sleep in populations with AUD. Although our analysis did not specifically measure reciprocal determinism through the interactions between self, behavior, and the environment, future studies in individuals with AUD should be designed to measure interventions that promote behavior change, including changes to optimize the environment and influence personal attitudes and beliefs about sleep. Additionally, the use of both objective (actigraphy) and subjective assessments of sleep is a strength. One methodological advantage was the use of sleep and symptom diaries to assess relapse. In our patient population, it is often difficult to obtain accurate information from the Timeline Follow-back assessment (post-discharge) for various reasons, including but not limited to losing patients to followup. For the participants who completed the study, the diaries provided a short-term mode of assessing relapse. Our study is not without limitations. A number of participants did not complete the outcome measures for various reasons, including not wanting to wear the Actiwatch and being lost to follow-up/ missing the follow-up "window." While much of the existing literature focuses on insomnia rates in populations with AUD, we did not measure insomnia and instead used a more broad measure of sleep disturbance (the PSQI). Our measure of "environment" (for sleep/recovery) was limited; although marital status emerged as an important factor in terms of relapse, it is not necessarily descriptive of the post-discharge environment (e.g., returning to a home, entering a structured living facility). We relied on self-report for assessing relapse, which is a limitation, but objective measures of relapse were beyond the scope of this study. Finally, the sample was not representative of all individuals with AUD seeking treatment and willing to participate in research; findings cannot be generalized.

\section{Conclusions/Future Directions}

Sleep-related beliefs and behaviors as well as sleep quality are important components of health-related quality of life, but may be particularly important among individuals with AUD. This is especially true when considering how best to support patients in their efforts to abstain from drinking across the transition from an inpatient treatment program back home and beyond. Results of the current analysis provide support for the SCT as a conceptual model in assessing cognitive and behavioral predictors of sleep quality and relapse among individuals with AUD with specific focus on the constructs of self-efficacy, outcome expectancies, and environment. Future research should consider novel methods of assessing both sleep disturbance/insomnia and relapse to drinking, as well as longer periods of follow-up. In larger datasets, it may be beneficial to distinguish between total sobriety and any or heavy drinking (potentially even treating relapse as a continuous variable - i.e., total or average number of drinks consumed) to allow for more nuanced data analyses. Since marital status emerged as an important variable in our analysis, future studies could assess quality/level of support provided by spouses (and other sources) post-discharge, in order to better understand outcomes. Based on our previous work regarding the importance of social support and our current results showing marital status as a predictor of relapse, we are interested in examining the effects of structured living (i.e., Oxford Houses or the like) vs. returning to the same pre-treatment environment, with the ultimate goal of targeted interventions based on risk. Other aspects of the postdischarge environment may also play an important role and should be explored.

\section{Compliance with Ethical Standards}

Conflict of Interest The authors declare that they have no conflict of interest.

Ethical Approval All procedures performed in studies involving human participants were in accordance with the ethical standards of the institutional and/or national research committee and with the 1964 Helsinki declaration and its later amendments or comparable ethical standards.

Welfare of Animals This article does not contain any studies with animals performed by any of the authors.

Informed Consent Informed consent was obtained from all individual participants included in the study.

Open Access This article is licensed under a Creative Commons Attribution 4.0 International License, which permits use, sharing, adaptation, distribution and reproduction in any medium or format, as long as you give appropriate credit to the original author(s) and the source, provide a link to the Creative Commons licence, and indicate if changes were made. The images or other third party material in this article are included in the article's Creative Commons licence, unless indicated otherwise in a credit line to the material. If material is not included in the article's Creative Commons licence and your intended use is not permitted by statutory regulation or exceeds the permitted use, you will need to obtain permission directly from the copyright holder. To view a copy of this licence, visit http://creativecommons.org/licenses/by/4.0/.

\section{References}

1. Kyung Lee, E. and A.B. Douglass, Sleep in psychiatric disorders: where are we now? Can J Psychiatr, 2010. 55(7): p. 403-412. 
2. WHO. Public health problems caused by harmful use of alcohol. (2013a) September 6, 2019]; Available from: https://www.who.int/ substance abuse/activities/public health_alcohol/en/.

3. WHO. (2013b). Alcohol (Fayetteville, N.Y.). Retrieved from https://www.who.int/substance_abuse/facts/alcohol/en/.

4. Angarita GA, Emadi N, Hodges S, Morgan PT. Sleep abnormalities associated with alcohol, cannabis, cocaine, and opiate use: a comprehensive review. Addiction science \& clinical practice. 2016;11(1):9-9.

5. Chakravorty S, Chaudhary NS, Brower KJ. Alcohol dependence and its relationship with insomnia and other sleep disorders. Alcohol Clin Exp Res. 2016;40(11):2271-82.

6. Zhabenko N, Wojnar M, Brower KJ. Prevalence and correlates of insomnia in a polish sample of alcohol-dependent patients. Alcohol Clin Exp Res. 2012;36(9):1600-7.

7. Ford DE, Kamerow DB. Epidemiologic study of sleep disturbances and psychiatric disorders. An opportunity for prevention? Jama. 1989;262(11):1479-84.

8. Fetting M. Perspectives on addiction: an integrative treatment model with clinical case studies. University of Southern California, USA: Sage Publications, Inc.; 2012.

9. Roehrs T, et al. Ethanol as a hypnotic in insomniacs: self administration and effects on sleep and mood. Neuropsychopharmacology. 1999;20(3):279-86.

10. Ebrahim IO, et al. Alcohol and sleep I: effects on normal sleep. Alcohol Clin Exp Res. 2013;37(4):539-49.

11. Thakkar, M.M., R. Sharma, and P. Sahota, Alcohol disrupts sleep homeostasis. Alcohol (Fayetteville, N.Y.), 2015. 49(4): p. 299-310.

12. Vitiello MV. Sleep, alcohol and alcohol abuse. Addict Biol. 1997;2(2):151-8.

13. Roehrs, T. and T. Roth, Insomnia as a path to alcoholism: tolerance development and dose escalation. Sleep, 2018. 41(8): p. zsy091.

14. Kolla BP, Schneekloth T, Mansukhani MP, Biernacka JM, HallFlavin D, Karpyak V, et al. The association between sleep disturbances and alcohol relapse: a 12-month observational cohort study. Am J Addict. 2015;24(4):362-7.

15. Brower KJ, Aldrich MS, Robinson EAR, Zucker RA, Greden JF. Insomnia, self-medication, and relapse to alcoholism. Am J Psychiatry. 2001;158(3):399-404.

16. Brooks AT, Krumlauf M, Fryer CS, Beck KH, Yang L, Ramchandani VA, et al. Critical transitions: a mixed methods examination of sleep from inpatient alcohol rehabilitation treatment to the community. PLoS One. 2016;11(8):e0161725.

17. Brower KJ, Perron BE. Sleep disturbance as a universal risk factor for relapse in addictions to psychoactive substances. Med Hypotheses. 2010;74(5):928-33.

18. Fatseas M, Kervran C, Auriacombe M. Sleep disorders and addictions: impact on quality of life and relapse vulnerability. Presse Med. 2016;45(12 Pt 1):1164-9.

19. Miller MB, Donahue ML, Carey KB, Scott-Sheldon LAJ. Insomnia treatment in the context of alcohol use disorder: a systematic review and meta-analysis. Drug Alcohol Depend. 2017;181:200-7.

20. Smith N, Hill R, Marshall J, Keaney F, Wanigaratne S. Sleep related beliefs and their association with alcohol relapse following residential alcohol detoxification treatment. Behav Cogn Psychother. 2014;42(5):593-604.

21. Brower KJ, Perron BE. Prevalence and correlates of withdrawalrelated insomnia among adults with alcohol dependence: results from a national survey. Am J Addict. 2010;19(3):238-44.

22. Brower KJ. Insomnia, alcoholism and relapse. Sleep Med Rev. 2003;7(6):523-39

23. Eslami AA, et al. Social cognitive theory as a theoretical framework to predict sustained abstinence 6 months after substance use treatment. J Subst Abus. 2018;23(3):300-6.
24. Bandura, A., Social foundations of thought and action: a social cognitive theory. 1986, Englewood Cliffs, NJ, US: Prentice-Hall, Inc. xiii, 617-xiii, 617.

25. Mead, M.P. and L.A. Irish, Application of health behaviour theory to sleep health improvement. J Sleep Res, 2019: p. e12950.

26. Harvey AG. A cognitive model of insomnia. Behav Res Ther. 2002;40(8):869-93.

27. Yang, C.M., A.J. Spielman, and P. Glovinsky, Nonpharmacologic strategies in the management of insomnia. Psychiatr Clin North Am, 2006. 29(4): p. 895-919; abstract viii.

28. Brooks AT, Magaña Lòpez M, Ranucci A, Krumlauf M, Wallen GR. A qualitative exploration of social support during treatment for severe alcohol use disorder and recovery. Addict Behav Rep. 2017;6:76-82

29. First, M.B., et al., Structured clinical interview for DSM-IV-TR axis I disorders, research version, patient edition. 2002, SCID-I/P New York, NY.

30. Sobell, L. and M. Sobell, Timeline follow-back: a technique for assessing self-reported alcohol consumption. Measuring Alcohol Consumption: Psychosocial and Biochemical Methods, 1992: p. 41-72.

31. Sobell, L.C. and M.B. Sobell, Timeline followback: user's guide 1996: Addiction Research Foundation= Fondation de la recherche sur la toxicomanie.

32. Flannery BA, Volpicelli JR, Pettinati HM. Psychometric properties of the Penn Alcohol Craving Scale. Alcohol Clin Exp Res. 1999;23(8):1289-95.

33. Sullivan JT, et al. Assessment of alcohol withdrawal: the revised clinical institute withdrawal assessment for alcohol scale (CIWAAr). Br J Addict. 1989;84(11):1353-7.

34. Åsberg M, Montgomery SA, Perris C, Schalling D, Sedvall G. A comprehensive psychopathological rating scale. Acta Psychiatr Scand. 1978;57(S271):5-27.

35. Tyrer P, Owen RT, Cicchetti DV. The brief scale for anxiety: a subdivision of the comprehensive psychopathological rating scale. J Neurol Neurosurg Psychiatry. 1984;47(9):970-5.

36. Montgomery SA, Åsberg M. A new depression scale designed to be sensitive to change. Br J Psychiatry. 1979;134(4):382-9.

37. Backhaus J, Junghanns K, Broocks A, Riemann D, Hohagen F. Test-retest reliability and validity of the Pittsburgh Sleep Quality Index in primary insomnia. J Psychosom Res. 2002;53(3):737-40.

38. Buysse DJ, Reynolds CF III, Monk TH, Berman SR, Kupfer DJ. The Pittsburgh Sleep Quality Index: a new instrument for psychiatric practice and research. Psychiatry Res. 1989;28(2):193-213.

39. Doi Y, Minowa M, Uchiyama M, Okawa M, Kim K, Shibui K, et al. Psychometric assessment of subjective sleep quality using the Japanese version of the Pittsburgh Sleep Quality Index (PSQI-J) in psychiatric disordered and control subjects. Psychiatry Res. 2000;97(2-3):165-72.

40. Johns MW. A new method for measuring daytime sleepiness: the Epworth sleepiness scale. Sleep. 1991;14(6):540-5.

41. Spira AP, et al. Reliability and validity of the Pittsburgh Sleep Quality Index and the Epworth Sleepiness Scale in older men. The Journals of Gerontology: Series A. 2011;67A(4):433-9.

42. Johns MW. Reliability and factor analysis of the Epworth Sleepiness Scale. Sleep. 1992;15(4):376-81.

43. John M. Daytime sleepiness, snoring, and obstructive sleep apnea. Chest. 1993;103:30-6.

44. Morin CM, Vallieres A, Ivers H. Dysfunctional beliefs and attitudes about sleep (DBAS): validation of a brief version (DBAS-16). Sleep. 2007;30(11):1547-54

45. Edinger JD, Wohlgemuth WK, Radtke RA, Coffman CJ, Carney CE. Dose-response effects of cognitive-behavioral insomnia therapy: a randomized clinical trial. Sleep. 2007;30(2):203-12. 
46. Fichten CS, Libman E, Creti L, Amsel R, Sabourin S, Brender W, et al. Role of thoughts during nocturnal awake times in the insomnia experience of older adults. Cogn Ther Res. 2001;25(6):665-92.

47. Rutledge CM, La Guardia AC, Bluestein D. Predictors of selfefficacy for sleep in primary care. J Clin Nurs. 2013;22(9-10): 1254-61.

48. Ree MJ, Harvey AG. Investigating safety behaviours in insomnia: the development of the Sleep-related Behaviours Questionnaire (SRBQ). Behav Chang. 2004;21(1):26-36.

49. Kushida CA, Chang A, Gadkary C, Guilleminault C, Carrillo O, Dement WC. Comparison of actigraphic, polysomnographic, and subjective assessment of sleep parameters in sleep-disordered patients. Sleep Med. 2001;2(5):389-96.

50. Paquet J, Kawinska A, Carrier J. Wake detection capacity of actigraphy during sleep. Sleep. 2007;30(10):1362-9.
51. Yavuz-Kodat, E., et al., Validity of actigraphy compared to polysomnography for sleep assessment in children with autism spectrum disorder. Frontiers in Psychiatry, 2019. 10(551).

52. Alling C, Balldin J, Bokström K, Gottfries CG, Karlsson I, Långström G. Studies on duration of a late recovery period after chronic abuse of ethanol: a cross-sectional study of biochemical and psychiatric indicators. Acta Psychiatr Scand. 1982;66(5):38497.

53. Brooks AT, Tuason RT, Chakravorty S, Raju S, Ritterband LM, Thorndike FP, et al. Online cognitive behavioral therapy for insomnia (CBT-I) for the treatment of insomnia among individuals with alcohol use disorder: study protocol for a randomized controlled trial. Pilot Feasibility Stud. 2018;4:183.

Publisher's Note Springer Nature remains neutral with regard to jurisdictional claims in published maps and institutional affiliations. 